\title{
Editorial: Antiplatelet Agents in Stroke Prevention
}

\author{
Gergely Feher ${ }^{1 *}$, David Hargroves ${ }^{2}$, Zsolt Illes ${ }^{3}$, Peter Klivenyi ${ }^{4}$, Liping Liu ${ }^{5}$ and \\ Laszlo Szapary 6
}

${ }^{1}$ Centre for Occupational Medicine, Medical School, University of Pécs, Pécs, Hungary, ${ }^{2}$ East Kent Hospitals University NHS Foundation Trust, Ashford, United Kingdom, ${ }^{3}$ Department of Neurology, Odense University Hospital, Odense, Denmark,

${ }^{4}$ Department of Neurology, Faculty of Medicine, Albert Szent-Györgyi Clinical Center, University of Szeged, Szeged, Hungary,

${ }^{5}$ Beijing Tiantan Hospital, Capital Medical University, Beijing, China, ${ }^{6}$ Medical School, University of Pécs, Pécs, Hungary

Keywords: stroke, antiplatelet agent, resistance, biomarker, outcome

\section{Editorial on the Research Topic}

\section{Antiplatelet Agents in Stroke Prevention}

Stroke is the leading cause of disability and the second most common cause of death worldwide based on the results of the Global Burden of Diseases Study (1). More than 80\% of all stroke syndromes are ischemic infarcts and their prevalence and cost will undoubtedly rise as aging populations increase (2). Despite extensive risk factor stratification and enhanced brain imaging, the etiology of stroke is still unknown in a significant proportion of patients. However, atherosclerosis, which is a low-grade inflammatory condition with detectable biomarkers, is the most likely culprit in most strokes (3).

Platelets play an essential role in the pathogenesis of atherothrombotic cardio- and cerebrovascular events, thus justifying the use of antiplatelet agents in their prevention. In their mini review, Valis and his workgroup summarized the evidence-based role of antiplatelet agents in the secondary prevention of non-cardioembolic stroke including aspirin, clopidogrel, dual antiplatelet therapy, and alternative agents such as cilostazol and ticagrelor Vališ et al.

Despite their efficacy, patients on these medications continue to suffer complications, which raises the possibility of the so-called "antiplatelet resistance" that is used to refer to the inability to protect individuals from thrombotic events (4). Due to the lack of standard methodology and randomized trials involving cerebrovascular patients, the clinical significance of antiplatelet resistance is contradictory (5). However, observational studies have shown an increased rate of ischemic cerebrovascular events in patients with high on-treatment of platelet reactivity (HPR) (so called resistance) in patients with both single (SAPT) and dual antiplatelet therapy (DAPT) (6).

Kang et al. analyzed the risk factors of clopidogrel resistance in patients taking mono- and dual therapy Kang et al. They demonstrated that HPR is more frequent in recurrent stroke patients receiving clopidogrel SAPT than in those receiving DAPT, and its risk factors may differ. The rates of HPR and clopidogrel resistance were lower in current smokers, which is rather surprising as smoking is one of the most important risk factors of atherosclerotic diseases. The role of smokers' paradox is not well-understood and merits further investigation.

In their paper Schrick et al. presented a modified platelet function test (mPFT) wherein they not only tested whole blood (WB), but also analyzed 1-h gravity sedimentation of the separated upper (UB) and lower half blood (LB) samples using Multiplate Analyzer to detect HPR as well as neutrophil antisedimentation rate (NAR) Shrick et al. This pilot study suggested that upward motion of platelets might be associated with increased thrombotic tendency.

It is worth noting that assessment of response to aspirin, GPI-s, or PAR-inhibitors is clinically not established as suggested by the Working Group on Thrombosis of the European Society of Cardiology (7). The most reliable, clinically best validated, and most widely used assays measured 
the effect of P2Y12-inhibitors (clopidogrel or prasugrel) and the recommended techniques were VASP-P assay, the VerifyNow device, and the Multiplate analyzer $(7,8)$. However, the routine use of platelet function testing is still not recommended $(7,8)$.

HPR can be associated with more ischemic events and recent studies have shown an increased bleeding risk in patients with low platelet reactivity (LPR) (9). Rosafio et al. also presented an interesting clinical case scenario of an aspirin ultra-responder patient Rosafio et al.

Since the coagulation system plays an important role in stroke pathogenesis, blood biomarkers of coagulation, and inflammation might render the possibility to differentiate which patients are at risk of poor clinical outcome. The ability to predict clinical outcome after an ischemic stroke may help to improve the selection of the most appropriate therapy (10). Based on recent studies, hemostatic changes during acute stroke in relation to antiplatelet resistance may predict the severity of an ischemic stroke.

In their in-depth review, Alhazzani et al. summarized the integration of specific biomarkers, genotype-, as well as phenotype-related data in antiplatelet therapy stratification in patients with acute ischemic stroke, which could be of great clinical impact on outcome Alhazzani et al.

Platelet endothelial aggregation receptor-1 (PEAR1) rs12041331 has been reported to affect agonist-stimulated platelet aggregation which can be associated with HPR in aspirin and clopidogrel treated patients, increasing the risk of unfavorable outcome. An observational Chinese study conducted by Zhang et al. could not confirm its role either in ischemic nor in bleeding events in TIA or minor stroke patients taking DAPT, doubting its prognostic value Zhang et al.

There is no doubt that taking antiplatelet agents or anticoagulants increases the risk of bleeding complications. Antiplatelet (especially DAPT) pretreatment potentially increases the risk of intracranial bleeding in thrombolyis/thrombectomy situations as well as in patients with traumatic brain injuries $(11,12)$. The potential harmful effects of DAPT have also been confirmed in this issue by the research of Lin et al. in more than 1,000 elderly patients with moderate to severe strokes who underwent systemic thrombolysis Lin et al. Although the patient cohorts were quite homogenous, the DAPT group contained relatively few patients ( $\sim 2 \%$ of the study cohort). Finally, based on a recent meta-analysis consisting of more than 60,000 patients, DAPT did not appear to be associated with a higher risk of adverse outcomes in thrombolyzed stroke patients, so dual pretreatment is not an indication to withdraw treatment, which is also confirmed by the authors (13).

Single small subcortical infarction (SSSI or lacunar stroke) accounts for $25 \%$ of all strokes and has heterogenous pathogenesis. Recent studies have shown an increased bleeding risk of SSSI patients, especially for those with underlying small vessel disease or taking DAPT (14). As the optimal treatment of these patients is not entirely clarified, Wang et al. analyzed the data of the CHANCE trial dividing patients into different subgroups based on antiplatelet treatment and SSSI etiology
Wang et al. They could not find any differences in the outcome of different subgroups, which merits further investigation.

Endovascular treatments have recently proven to be effective in improving functional outcomes for selected patients with large vessel occlusion, although it can cause injury to endothelial cells leading to activation of local platelet aggregation and subsequent early reocclusion, and therefore more effective and safe thrombolytic agents are required (15). Glycoprotein (GP) IIb-IIIa inhibitors are short-acting selective reversible antiplatelet agents widely used in acute coronary syndromes and have recently emerged as promising therapeutic agents for ischemic stroke management. Among them, tirofiban may be considered safe in low doses (15). Two studies focused on the efficacy and safety of tirofiban in relation to the management of large vessel occlusion (LVO) including thrombectomy. Huo et al. showed its beneficial effects in 650 ischemic stroke patients; based on their findings tirofiban was found to be associated with superior clinical outcomes in anterior circulation stroke and major stroke patients and had a trend to lower the risk of mortality at 90day follow-ups with no increase in bleeding rates compared to the non-tirofiban group Huo et al. In the other study presented by $\mathrm{Ma}$ et al. covering $\sim 200$ patients, no significant differences in safety and efficacy outcomes on successful recanalization, clinical improvement, or 3-month mRS could be found between the tirofiban and non-tirofiban groups Ma et al. The administration of tirofiban seems to be safe in LVO patients but its efficacy and safety merits further investigation.

Intracerebral hemorrhage $(\mathrm{ICH})$ may be caused by antiplatelet treatment and prior treatment may be associated with worse clinical outcomes; however, previous studies on $\mathrm{ICH}$ growth and outcome have found conflicting results $(16,17)$. In their meta-analysis of 31 studies, $\mathrm{Wu}$ et al. found no association with hematoma expansion or functional outcomes in $\mathrm{ICH}$ patients, but increased mortality rates raised the possibility of the introduction of early-time platelet function reversal strategies $\mathrm{Wu}$ et al. It is worth noting that the randomized PATCH trial found platelet transfusion to be inferior compared to standard care in ICH patients (18).

The rupture of an intracranial aneurysm could be a lifethreatening disease accounting for a relatively small but significant number of stroke syndromes. The role of prior antiplatelet use on the risk of bleeding and outcome is not well-studied. In their interesting meta-analysis covering nearly 9,000 participants, Yang et al. found that prior aspirin use was associated with a significantly lower risk of aneurysm growth and rupture, suggesting the potential protective effect of aspirin Yang et al. However, it is not well-understood and merits further investigations.

\section{AUTHOR CONTRIBUTIONS}

This editorial was written by GF and checked by DH, ZI, PK, LL, and LS. All authors contributed to the article and approved the submitted version. 


\section{REFERENCES}

1. GBD 2019 Diseases and Injuries Collaborators. Global burden of 369 diseases and injuries in 204 countries and territories, 1990-2019: a systematic analysis for the Global Burden of Disease Study 2019. Lancet. (2020) 396:120422. doi: 10.1016/S0140-6736(20)30925-9

2. Xu W, Huang J, Yu Q, Yu H, Pu Y, Shi Q. A systematic review of the status and methodological considerations for estimating risk of first ever stroke in the general population. Neurol Sci. (2021) 42:223547. doi: 10.1007/s10072-021-05219-w

3. Banerjee C, Chimowitz MI. Stroke caused by atherosclerosis of the major intracranial arteries. Circ Res. (2017) 120:50213. doi: 10.1161/CIRCRESAHA.116.308441

4. Feher G, Feher A, Pusch G, Koltai K, Tibold A, Gasztonyi B, et al. Clinical importance of aspirin and clopidogrel resistance. World J Cardiol. (2010) 2:171-86. doi: 10.4330/wjc.v2.i7.171

5. Koltai K, Kesmarky G, Feher G, Tibold A, Toth K. Platelet aggregometry testing: molecular mechanisms, techniques and clinical implications. Int J Mol Sci. (2017) 18:1803. doi: 10.3390/ijms18081803

6. Fiolaki A, Katsanos AH, Kyritsis AP, Papadaki S, Kosmidou M, Moschonas IC, et al. High on treatment platelet reactivity to aspirin and clopidogrel in ischemic stroke: a systematic review and meta-analysis. J Neurol Sci. (2017) 376:112-16. doi: 10.1016/j.jns.2017.03.010

7. Aradi D, Collet JP, Mair J, Plebani M, Merkely B, Jaffe AS, et al. Platelet function testing in acute cardiac care - is there a role for prediction or prevention of stent thrombosis and bleeding? Thromb Haemost. (2015) 113:221-30. doi: 10.1160/TH14-05-0449

8. Sibbing D, Aradi D, Alexopoulos D, Ten Berg J, Bhatt DL, Bonello L, et al. Updated expert consensus statement on platelet function and genetic testing for guiding $\mathrm{P}_{2} \mathrm{Y}_{12}$ receptor inhibitor treatment in percutaneous coronary intervention. JACC Cardiovasc Interv. (2019) 12:152137. doi: 10.1016/j.jcin.2019.03.034

9. Aradi D, Gross L, Trenk D, Geisler T, Merkely B, Kiss RG, et al. Platelet reactivity and clinical outcomes in acute coronary syndrome patients treated with prasugrel and clopidogrel: a pre-specified exploratory analysis from the TROPICAL-ACS trial. Eur Heart J. (2019) 40:194251. doi: 10.1093/eurheartj/ehz202

10. Csecsei P, Várnai R, Nagy L, Kéki S, Molnár T, Illés $Z$, et al. Larginine pathway metabolites can discriminate paroxysmal from permanent atrial fibrillation in acute ischemic stroke. Ideggyogy Sz. (2019) 72:7988. doi: 10.18071/isz.72.0079

11. Nguyen KA, Eadon MT, Yoo R, Milway E, Kenneally A, Fekete K, et al. Risk factors for bleeding and clinical ineffectiveness associated with clopidogrel therapy: a comprehensive meta-analysis. Clin Transl Sci. (2021) 14:64555. doi: $10.1111 /$ cts. 12926
12. Tsivgoulis G, Katsanos AH, Zand R, Sharma VK, Köhrmann M, Giannopoulos $\mathrm{S}$, et al. Antiplatelet pretreatment and outcomes in intravenous thrombolysis for stroke: a systematic review and meta-analysis. J Neurol. (2017) 264:122735. doi: 10.1007/s00415-017-8520-1

13. Malhotra K, Katsanos AH, Goyal N, Ahmed N, Strbian D, Palaiodimou L, et al. Safety and efficacy of dual antiplatelet pretreatment in patients with ischemic stroke treated with IV thrombolysis: a systematic review and metaanalysis. Neurology. (2020) 94:e657-66. doi: 10.1212/WNL.000000000000 8961

14. Tsai HH, Kim JS, Jouvent E, Gurol ME. Updates on prevention of hemorrhagic and lacunar strokes. J Stroke. (2018) 20:167-79. doi: 10.5853/jos.2018.00787

15. Zhu X, Cao G. Safety of glycoprotein IIb-IIIa inhibitors used in stroke-related treatment: a systematic review and meta-analysis. Clin Appl Thromb Hemost. (2020) 26:1076029620942594. doi: 10.1177/1076029620942594

16. Bakheet MF, Pearce LA, Hart RG. Effect of addition of clopidogrel to aspirin on subdural hematoma: meta-analysis of randomized clinical trials. Int $J$ Stroke. (2015) 10:501-5. doi: 10.1111/ijs.12419

17. Steiner T, Al-Shahi Salman R, Beer R, Christensen H, Cordonnier C, Csiba L, et al. European Stroke Organisation (ESO) guidelines for the management of spontaneous intracerebral hemorrhage. Int J Stroke. (2014) 9:840-55. doi: 10.1111/ijs.12309

18. Baharoglu MI, Cordonnier C, Al-Shahi Salman R, de Gans K, Koopman MM, Brand A, et al. Platelet transfusion versus standard care after acute stroke due to spontaneous cerebral haemorrhage associated with antiplatelet therapy (PATCH): a randomised, open-label, phase 3 trial. Lancet. (2016) 387:2605-13. doi: 10.1016/S0140-6736(16)30392-0

Conflict of Interest: The authors declare that the research was conducted in the absence of any commercial or financial relationships that could be construed as a potential conflict of interest.

Publisher's Note: All claims expressed in this article are solely those of the authors and do not necessarily represent those of their affiliated organizations, or those of the publisher, the editors and the reviewers. Any product that may be evaluated in this article, or claim that may be made by its manufacturer, is not guaranteed or endorsed by the publisher.

Copyright (c) 2021 Feher, Hargroves, Illes, Klivenyi, Liu and Szapary. This is an open-access article distributed under the terms of the Creative Commons Attribution License (CC BY). The use, distribution or reproduction in other forums is permitted, provided the original author(s) and the copyright owner(s) are credited and that the original publication in this journal is cited, in accordance with accepted academic practice. No use, distribution or reproduction is permitted which does not comply with these terms. 